\title{
Feasibility of functional electrical stimulation for control of seated posture after spinal cord injury: A simulation study
}

\author{
Ari J. Wilkenfeld, MD, PhD; ${ }^{1-2 *}$ Musa L. Audu, PhD; ${ }^{3}$ Ronald J. Triolo, $\mathbf{P h D}^{\mathbf{2 - 4}}$ \\ ${ }^{1}$ University Hospitals of Cleveland, The Case Medical Center, Cleveland, OH; ${ }^{2}$ Department of Veterans Affairs (VA) \\ Motion Study Laboratory, Louis Stokes Cleveland VA Medical Center, Cleveland, OH; Departments of ${ }^{3}$ Biomedical \\ Engineering and ${ }^{4}$ Orthopaedics, Case Western Reserve University School of Medicine, Cleveland, $\mathrm{OH}$
}

\begin{abstract}
We performed this study to determine the feasibility of controlling and stabilizing seated posture with functional electrical stimulation (FES) after paralysis from spinal cord injury (SCI) using computer simulations and a 3-dimensional model of the hip and trunk. We used the model to approximate the range of postures in the sagittal and transverse planes attainable by a seated subject and to estimate the maximum restorative moment that could be produced in a neutral posture in response to a disturbance. The simulations predicted that approximately 28 degrees of forward flexion in the sagittal plane (combined hip and trunk) and 9 degrees of lateral bending in the transverse plane should be possible with FES and that a maximum disturbance rejection moment of approximately 45 newton meters could be expected with the chosen muscle set. We tested a subject with a motor complete thoracic SCI and implanted electrodes in a subset of the selected muscles to compare the moments the subject required to maintain various hip and trunk positions with those predicted by the model. Although a significant range of seated postures was possible with FES, the data demonstrated that more complete activation of the paralyzed muscles would be needed for the subject to fully achieve the theoretical range of motion. With further refinements, we could apply these techniques to the design of control systems for regulation of seated posture and dynamic motion of the torso.
\end{abstract}

Key words: biomechanics, functional electrical stimulation, maximum force output, moment of force, musculoskeletal model, posture, rehabilitation, seating, spinal cord injury, trunk muscles.

\section{INTRODUCTION}

Paralysis as a result of spinal cord injury (SCI) can severely compromise an individual's ability to control the movements of the limbs and trunk. Functional electrical stimulation (FES) has a long history as a rehabilitation tool for restoring mobility after SCI. While much research has been directed toward providing individuals with cervical or thoracic ( $\mathrm{T}$ ) level injuries with the ability to grasp, reach, stand, transfer, and step with the application of FES [1-5], relatively little attention has been given the potentially useful and clinically relevant issue of control of seated posture.

The lack of voluntary control of the trunk muscles after SCI can have serious functional and health-related implications. The inability to stabilize the spine and pelvis renders those with paralysis unable to vary seated posture or maintain anything other than one passive

\footnotetext{
Abbreviations: 3-D = 3-dimensional, $d f=$ degrees of freedom, FES = functional electrical stimulation, $\mathrm{L}=$ lumbar, $\mathrm{S}=$ sacral, SCI = spinal cord injury, SIMM = Software for Musculoskeletal Modeling, $\mathrm{T}=$ thoracic, VA = Department of Veterans Affairs.

*Address all correspondence to Ari J. Wilkenfeld, MD, PhD; Motion Study Laboratory-151A, Louis Stokes Cleveland Department of Veterans Affairs Medical Center, 10701 East Blvd, Cleveland, $\mathrm{OH}$ 44106; 440-473-2408; fax: 216-231-3433.

Email: awilkenfeld@alum.mit.edu

DOI: 10.1682/JRRD.2005.06.0101
} 
stable position, which can result in the adoption of unhealthy seated postures for prolonged periods of time. This can lead to skeletal deformities, increased risk for development of decubitus ulcers, and imposition of large and nonphysiological pressure on internal organs that may compromise their function [6-7]. Furthermore, paralysis of the hip and spinal muscles can dramatically decrease workspace and limit an individual's ability to interact with the environment [8]. Wheelchair users often rely exclusively on unilateral reach and adopt various compensatory strategies to substitute for active trunk stability, such as leaning away from the object being grasped to passively balance the imposed moments, holding on to the wheel rim, or crooking the elbow under the push handle with one arm while reaching with the other, which further limits the available workspace. Bilateral reach is often impossible.

Clinical experience with the joint Case Western Reserve University and Department of Veterans Affairs (VA) implanted neuroprosthesis [9] indicates that activation of the paralyzed hip and trunk extensor muscles allows different stable equilibrium positions in the seated subject with motor complete paraplegia [10]. Electrodes implanted at the T12 to first lumbar (L) spinal roots for recruitment of the $L$ erector spinae and stabilization of the trunk over the pelvis improve standing postures; for seated postures, they may also expand the bilateral workspace of subjects seated without a backrest, provide a more natural anterior pelvic tilt and L curve, and extend forward reach by up to $8 \mathrm{~cm}$. We hypothesized that additional activation of the hip extensor muscles (gluteus maximus and semimembranosus) would likely further improve seated reaching performance [11].

We performed this study to examine the feasibility of controlling seated posture in individuals with SCI by stimulating the hip and trunk musculature. The potential for controlling pelvis and trunk position with FES was analyzed with computer simulations and a 3-dimensional (3D) model that included articulations of the $\mathrm{L}$ spine and pelvis, as well as the erector spinae, rectus abdominis, quadratus lumborum, gluteus maximus, rectus femoris, and semimembranosus muscles. We took values for normal anatomy and force production from the literature and modified them with previously established standards for force output expected from electrically stimulated paralyzed muscle. We hypothesized that activating the muscles of the torso and hips with FES would provide stability over a clinically relevant set of seated postures and thus allow subjects to manipulate objects in the environment without leaning over or using their other hand for support.

\section{METHODS}

A 3-D biomechanical model of the trunk of a nondisabled person that incorporated the musculoskeletal structures of the trunk and pelvis was developed. We approximated the inertial parameters for the model by using standardized data for a human male and making geometric assumptions for the different body segments. For the experimental portion of the project, the inertial parameters were reformulated based on measurements of our subject with SCI. The muscle insertion points and muscle cross-sectional areas were taken from the literature and based on original, previously reported work in our laboratory. Maximum force output of the muscles per unit of cross-sectional area was determined for the model and based on maximum voluntary back-extension moments reported in the literature. We then scaled these maximum force outputs to represent the expected output, based on previous data, from an electrically stimulated paralyzed muscle. All muscles in this model were represented as simple force generators where force output was proportional to activation level. We also conducted an experiment with a subject with SCI to approximate the muscle volume activated by the current FES system and compare required joint moments calculated from the experiment with muscle outputs predicted by the model. The subject who participated in this experimental procedure signed informed consent documents approved by the institutional review board of the Louis Stokes Cleveland VA Medical Center. The model was implemented with the Software for Interactive Musculoskeletal Modeling (SIMM) package from MusculoGraphics, Inc, Santa Rosa, California (http://www.musculographics.com), which includes graphical representations and routines for calculating muscle-moment arms given different postures. We used the SD-FAST dynamic engine (Parametric Technology Corporation, Needham, Massachusetts) to generate dynamic routines, including procedures for calculating the necessary moment for a given posture (inverse dynamics), and to apply external forces and moments to the model.

\section{Model Development}

\section{Kinematic Constraints}

The trunk component of the model focused on the actions of the $\mathrm{L}$ vertebrae, which are responsible for a majority of spinal movement in flexion-extension and lateral bending. The $\mathrm{T}$ spine was assumed rigid, which 
represented a spinal fusion or other stabilizing instrumentation common in people with SCI.

The $\mathrm{L}$ spine has six joints, each with 3 degrees of freedom ( $d f$ ). To be able to individually control all $18 d f$ and achieve any arbitrary posture, an individual would require a large number of independent muscle fascicles. An example of such an approach is the model of the $\mathrm{L}$ spine developed by Cholewicki and McGill [12], which incorporated 90 independently controlled muscle fascicles and preserved all $18 d f$. While some researchers maintain that this level of independent activation of the trunk musculature is needed for control of spinal position [13], others contend that no experimental evidence exists of the wide discrepancy in activation of different backmuscle fascicles that one would expect from the activation patterns predicted by such models [14]. For this study, we assumed that the trunk could be controlled with a relatively small number of independent actuators that represented discrete muscles (quadratus lumborum and rectus abdominis) or muscle groups (columns of the erector spinae) that worked in synergy if certain kinematic constraints were enforced. Our resulting formulation assumed that all moments acting on the $\mathrm{L}$ spine resulted in static equilibrium about the L5 to first sacral (S) joint (where such moments would be the highest), while total flexion-extension (pitch) and lateral bending (roll) were distributed across all L intervertebral segments in fixed proportions. Axial rotation about the spine (yaw) was set to zero for these simulations, and the muscles were required to output forces to keep the spine in this neutral posture against applied loads and gravity. Thus, although moments were only balanced about the L5-S1 joint, the intervertebral angles for all the other L segments $(\alpha)$ were constrained to constant ratios of the L5-S1 joint. These ratios were proportional to the given joint's range of motion as originally measured by White and Panjabi [15]. Our technique is similar to that for distributing the total bend of the spine to its segments used by McGill [16]. These constraints are described in

$\mathrm{L}$ segment rotation (degrees) $i=\alpha_{i} \times$ total spine rotation (degrees),

where for flexion-extension:

$$
\begin{aligned}
& \alpha_{\mathrm{L} 5-\mathrm{S} 1}=19.8 \%, \alpha_{\mathrm{L} 4-\mathrm{L} 5}=18.6 \%, \alpha_{\mathrm{L} 3-\mathrm{L} 4}=17.5 \%, \\
& \alpha_{\mathrm{L} 2-\mathrm{L} 3}=16.3 \%, \alpha_{\mathrm{L} 1-\mathrm{L} 2}=13.9 \%, \text { and } \alpha_{\mathrm{T} 12-\mathrm{L} 1}=13.9 \% ;
\end{aligned}
$$

and for lateral bending:

$$
\begin{aligned}
& \alpha_{\mathrm{L} 5-\mathrm{S} 1}=8.1 \%, \alpha_{\mathrm{L} 4-\mathrm{L} 5}=16.2 \%, \alpha_{\mathrm{L} 3-\mathrm{L} 4}=21.6 \%, \\
& \alpha_{\mathrm{L} 2-\mathrm{L} 3}=16.2 \%, \alpha_{\mathrm{L} 1-\mathrm{L} 2}=16.2 \%, \text { and } \alpha_{\mathrm{T} 12-\mathrm{L} 1}=21.6 \% .
\end{aligned}
$$

We also constrained the pelvic and femoral components to simplify the computational structure of the biomechanical model. We assumed the femurs were fixed to the chair at a constant angle roughly parallel to the ground (and perpendicular to the gravity vector). As a result of this approximation, the model only allowed for pelvic flexion-extension and included neither axial rotation nor lateral bending of the pelvis. The full model, therefore, has $4 d f$ that must be controlled (flexionextension of the hip and flexion-extension, lateral bending, and axial rotation of the spine).

\section{Inertial Parameters}

The inertial parameters were calculated based on a standard male as defined by the 1998 U.S. Army Male Anthropomorphic Survey (http://ioe.engin.umich.edu/ ioe491/Data1/Ans88 m.pdf). The model we used for the computations was $1.75 \mathrm{~m}$ tall and weighed $78 \mathrm{~kg}$. We calculated the geometric properties of the pelvis by assuming it was a rectangular solid. The height of the pelvis was calculated as a percentage of the total height of the model as defined by Drillis and Contini (from Winter [17]). The relative width and depth of the pelvis were taken from the U.S. Army survey. The weight of the pelvis was calculated as a percentage of the subject's total weight as defined by Dempster (from Winter [17]). We obtained inertial parameters for the head, trunk, and upper limbs by the same method as for the pelvis, except we assumed that the upper limbs and trunk were cylindrical solids and the head was a sphere. The head and arms were constrained to move with the $\mathrm{T}$ segment. The inertial parameters we used are summarized in Table 1. For calculations involving the subject with SCI, we reformulated the intertial parameters of the model for his height and weight. The relative dimensions of each of his body segments were taken by direct measurements with calipers. The data for this version of the model are summarized in Table 2.

\section{Hip and Trunk Musculature and Passive Moment}

The bilateral erector spinae (lumped iliocostalis and longissimus), quadratus lumborum, and rectus abdominis were included in this model because these muscles are either currently implanted in FES subjects, as in the case of the erector spinae [9,18-19], or accessible via surface stimulation and possible targets for future implantation $[18,20-23]$. The quadratus lumborum was chosen because of its large moment arm for lateral bending and 
Table 1.

Inertial parameters for male pelvis-trunk model. Coordinate system was defined such that $Y$ was upward, $X$ was forward, and $Z$ (positive) was to model's right. Note that origin of proximal joints of arms was $0.2 \mathrm{~m}$ to right and left of top of torso.

\begin{tabular}{|c|c|c|c|c|c|c|c|}
\hline \multirow[t]{2}{*}{ Parameter } & \multirow{2}{*}{$\begin{array}{l}\text { Height } \\
\text { (m) }\end{array}$} & \multirow{2}{*}{$\begin{array}{l}\text { Weight } \\
\text { (kg) }\end{array}$} & \multicolumn{2}{|c|}{ Geometric Approximation } & \multicolumn{3}{|c|}{$\begin{array}{l}\text { Center Mass Location from } \\
\text { Proximal End of Joint }\end{array}$} \\
\hline & & & Major Axis & Minor Axis & $Y(\mathbf{m})$ & $X(\mathbf{m})$ & $Z(\mathrm{~m})$ \\
\hline Subject & 1.75 & 78.0 & - & - & - & - & - \\
\hline Pelvis & 0.08 & 8.3 & 0.34 & 0.24 & 0.04 & 0.06 & 0.00 \\
\hline L5 & 0.03 & 2.0 & 0.31 & 0.23 & 0.02 & 0.05 & 0.00 \\
\hline L4 & 0.03 & 2.1 & 0.31 & 0.23 & 0.02 & 0.03 & 0.00 \\
\hline L3 & 0.04 & 2.2 & 0.31 & 0.23 & 0.02 & 0.03 & 0.00 \\
\hline $\mathrm{L} 2$ & 0.03 & 1.8 & 0.31 & 0.23 & 0.01 & 0.04 & 0.00 \\
\hline L1 & 0.03 & 1.8 & 0.31 & 0.23 & 0.01 & 0.05 & 0.00 \\
\hline Thoracic Torso & 0.34 & 20.6 & 0.32 & 0.24 & 0.17 & 0.06 & 0.00 \\
\hline Head & 0.22 & 6.3 & 0.18 & 0.18 & 0.11 & 0.00 & 0.00 \\
\hline Right Arm & 0.58 & 3.9 & 0.09 & 0.08 & -0.29 & 0.00 & 0.00 \\
\hline Left Arm & 0.58 & 3.9 & 0.09 & 0.08 & -0.29 & 0.00 & 0.00 \\
\hline
\end{tabular}

Table 2.

Inertial parameters for pelvis-trunk model modified to fit subject with spinal cord injury. Coordinate system was defined such that $Y$ was upward, $X$ was forward, and $Z$ (positive) was to model's right. Note that origin of proximal joints of arms was $0.2 \mathrm{~m}$ to right and left of top of torso.

\begin{tabular}{|c|c|c|c|c|c|c|c|}
\hline \multirow{2}{*}{ Parameter } & \multirow{2}{*}{$\begin{array}{l}\text { Height } \\
\text { (m) }\end{array}$} & \multirow{2}{*}{$\begin{array}{l}\text { Weight } \\
\text { (kg) }\end{array}$} & \multicolumn{2}{|c|}{ Geometric Approximation } & \multicolumn{3}{|c|}{$\begin{array}{l}\text { Center Mass Location from } \\
\text { Proximal End of Joint }\end{array}$} \\
\hline & & & Major Axis & Minor Axis & $Y(\mathbf{m})$ & $X(\mathbf{m})$ & $Z(\mathbf{m})$ \\
\hline Subject & 1.73 & 93.3 & - & - & - & - & - \\
\hline Pelvis & 0.09 & 10.0 & 0.37 & 0.26 & 0.04 & 0.04 & 0.00 \\
\hline L5 & 0.03 & 2.4 & 0.37 & 0.30 & 0.02 & 0.05 & 0.00 \\
\hline L4 & 0.03 & 2.5 & 0.37 & 0.30 & 0.02 & 0.05 & 0.00 \\
\hline L3 & 0.04 & 2.6 & 0.37 & 0.30 & 0.02 & 0.05 & 0.00 \\
\hline L2 & 0.03 & 2.1 & 0.37 & 0.30 & 0.01 & 0.05 & 0.00 \\
\hline L1 & 0.03 & 2.1 & 0.37 & 0.30 & 0.01 & 0.06 & 0.00 \\
\hline Thoracic Torso & 0.34 & 24.6 & 0.39 & 0.21 & 0.17 & 0.02 & 0.00 \\
\hline Head & 0.22 & 7.6 & 0.18 & 0.17 & 0.11 & 0.00 & 0.00 \\
\hline Right Arm & 0.58 & 4.7 & 0.08 & 0.09 & -0.29 & 0.00 & 0.00 \\
\hline Left Arm & 0.58 & 4.7 & 0.08 & 0.09 & -0.29 & 0.00 & 0.00 \\
\hline
\end{tabular}

the rectus abdominis for trunk flexion moment. Insertion points for these muscles (Figure 1) were taken from cadaver studies previously published by our laboratory [24]. These parameters are summarized in Table 3. Cross-sectional areas for these muscles were taken from the imaging study by Tracy et al. [25].

A wide variety of values are given in the literature for the maximum force per cross-sectional area of trunk muscle. Granata and Marras list a physiologically feasible range of 30-100 N/cm ${ }^{2}$ [26]. To determine a reasonable value for our trunk model, we took the maximum voluntary extension moment that can be generated by a nondisabled male in a neutral standing position $(125 \mathrm{~N} \cdot \mathrm{m}$ [27]) and divided by the moment arm and cross-sectional area of the muscle in that posture. This yielded a value of $78 \mathrm{~N} / \mathrm{cm}^{2}$. The passive moments at the lumbrosacral joint of the spinal column were taken from the review of the literature by White and Panjabi [15]. For flexion of the spine, they reported a $3^{\circ}$ neutral zone (no passive elasticity) followed by a passive elasticity of $1.0 \mathrm{~N} \cdot \mathrm{m} /{ }^{\circ}$ of flexion of the L5-S1 joint. For lateral bending of the spine, they reported a neutral zone of $1.8^{\circ}$ followed by a passive 
(a)

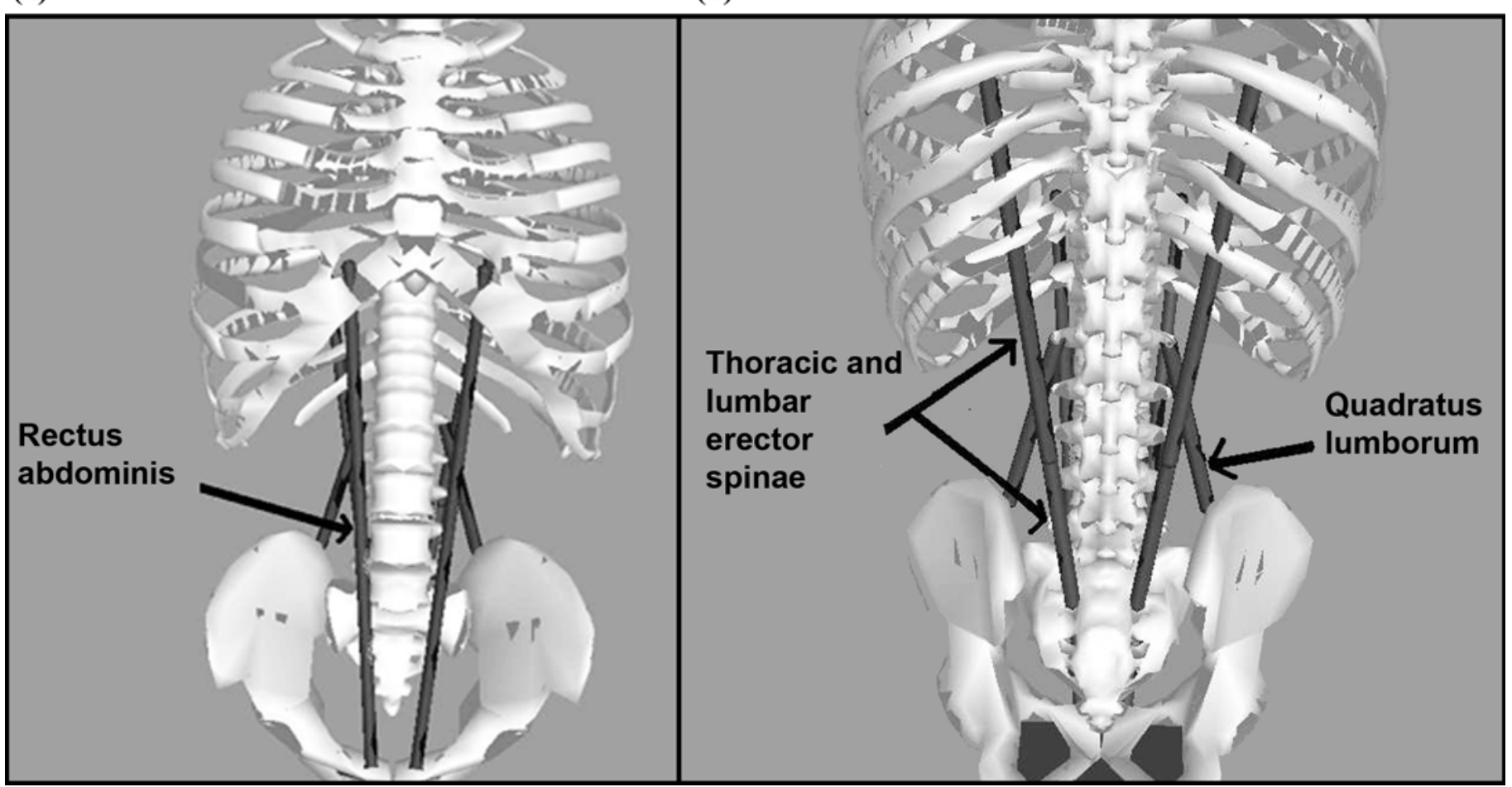

Figure 1.

Representation of trunk musculature included in model shown from (a) anterior and (b) posterior.

Table 3.

Length of trunk muscles.

\begin{tabular}{lc}
\hline \multicolumn{1}{c}{ Muscle } & Length $(\mathbf{c m})$ \\
\hline Erector Spinae & 30.0 \\
Rectus Abdominis & 36.0 \\
Quadratus Lumborum & 10.0 \\
\hline \hline
\end{tabular}

elasticity of $7.69 \mathrm{~N} \cdot \mathrm{m} /{ }^{\circ}$ of the L5-S1 joint. Passive moment in the direction of axial rotation about the spine did not come into play, since we constrained the muscles to prevent any movement in that direction for these simulations. Therefore, the passive moments included in the simulations were for spine flexion

L5-S1 joint angle $<1.0^{\circ}=0 \mathrm{~N} \cdot \mathrm{m}$ and

L5-S1 joint angle $>1.0^{\circ}=1.0 \mathrm{~N} \cdot \mathrm{m} /{ }^{\circ} \times\left(\right.$ L5-S1 joint angle $\left.-1.0^{\circ}\right)$,

and for spine lateral bending

L5-S1 joint angle $<1.8^{\circ}=0 \mathrm{~N} \cdot \mathrm{m}$ and

L5-S1 joint angle $>1.8^{\circ}=7.69 \mathrm{~N} \cdot \mathrm{m} /{ }^{\circ} \times\left(\right.$ L5 $-\mathrm{S} 1$ joint angle $\left.-1.8^{\circ}\right)$.
Previous experiments on lower-limb FES have shown that optimal recruitment can yield approximately 50 percent of the force available to a muscle in a nondisabled individual [28]. To predict the workspace and restorative force of a posture control system using FES, we adapted the nondisabled model such that maximum force output from each of the muscles would be 50 percent of their nondisabled values.

In the experiment with the subject with SCI, we set out to determine the actual force output of the electrically stimulated erector spinae muscle bulk innervated by the implanted electrodes. The volunteer with SCI was a male with a T6 motor and sensory complete (American Spinal Injury Association A classification) ${ }^{*}$ injury. The volunteer received an implanted FES system for exercise and standing approximately 15 months postinjury, in December

\footnotetext{
*American Spinal Injury Association has developed an impairment scale for neurological classification of spinal cord injury. The scale is based on test of key muscles and levels of dermatomes and describes intact sensory and motor level. It consists of five categories (A to E). A = complete injury with no motor or sensory response below injury level.
} 
2000, and was an experienced system user [9]. His FES system included electrodes inserted bilaterally at the T12L1 spinal roots for as much activation of the $\mathrm{L}$ erector spinae as possible, as well as epimysial electrodes sutured to the primary nerve entry points of the gluteus medius and semimembranosus bilaterally [19].

The subject was seated in a chair without a backrest in a safety harness in the measurement area of a Vicon 360 (Oxford Metrics, Oxford, United Kingdom) optical motion-capture system (Figure 2). We affixed reflective markers to the skin over the bony prominence of the T1 vertebral process, the anterior superior iliac spines (to estimate the location of the hip joints), and the sacrum. Using these markers, we were able to calculate the sagittal plane orientation of the pelvis (as estimated by the motion of the sacral marker about the hip joint centers) and the spine (as approximated by the motion of the T1 marker about the sacrum). All angles were measured relative to what they would be if the subject were standing upright, based on a reference trial.

The stimulated responses of the erector spinae and other muscles to the biphasic, charge-balanced pulses generated by the implant were calibrated via standard methods developed in our laboratory [18]. Stimulus amplitude and frequency [29] were fixed at $20 \mathrm{~mA}$ and $20 \mathrm{~Hz}$, respectively (values used in our laboratory based on experience that they simultaneously maximize force production characteristics and minimize the effects of fatigue). We varied pulse duration to determine the minimum value of pulse duration at which a stimulated contraction was first observed (threshold) and the maximum value of pulse duration above which no additional force was recruited or other muscles or undesired reflexes were activated (saturation). The threshold and saturation values define an idealized linear recruitment curve, offset, and the maximum values of the dynamic range of available input stimulus pulse durations (Figure 3). During the experimental sessions, we varied stimulus pulse duration as a percentage of the available range of values between threshold and saturation for each muscle, which corresponded roughly to the percentage of contractile output available from each electrode.

The posture of the subject was measured in three different conditions: a neutral posture with no electrical activation of the erector spinae, a stable posture achieved with pulse durations set at 50 percent of the effective dynamic range of the implanted erector spinae electrodes, and a stable posture achieved with stimulus values set at

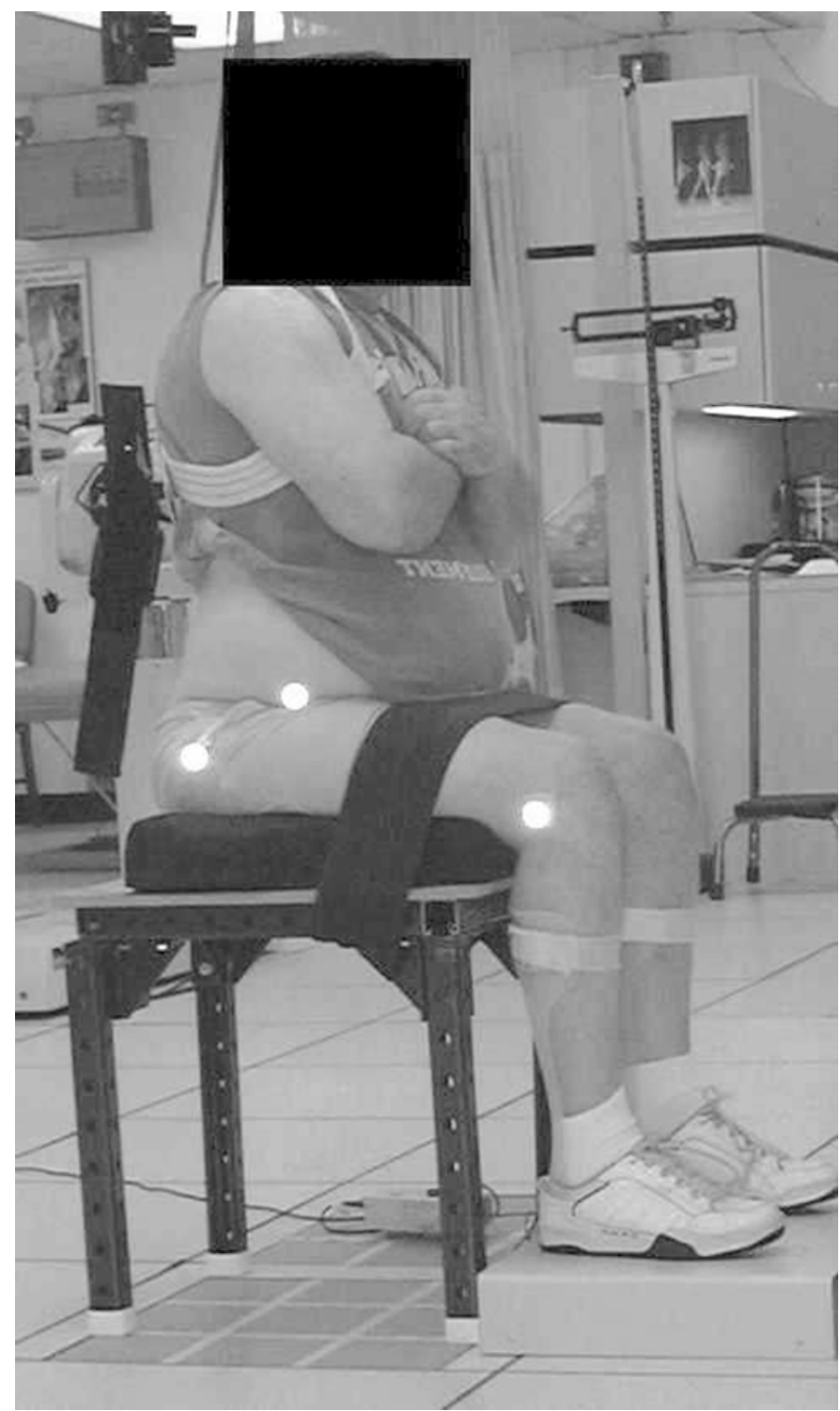

Figure 2.

Experimental setup for spinal cord injury motion-capture experiment. Markers are placed on bony landmarks as estimates of joint positions. Harness is kept loose but available for safety purposes.

100 percent of the effective input range of the implanted erector spinae electrodes (i.e., saturation). All three positions were measured four times (one data set for the $50 \%$ condition was unusable because of technical difficulties), with breaks of approximately 2 min between each trial and several longer breaks for pressure relief scattered throughout the experimental session. For each trial, the subject was assisted in leaning far backward or far forward before stimulation was applied (each set of three 


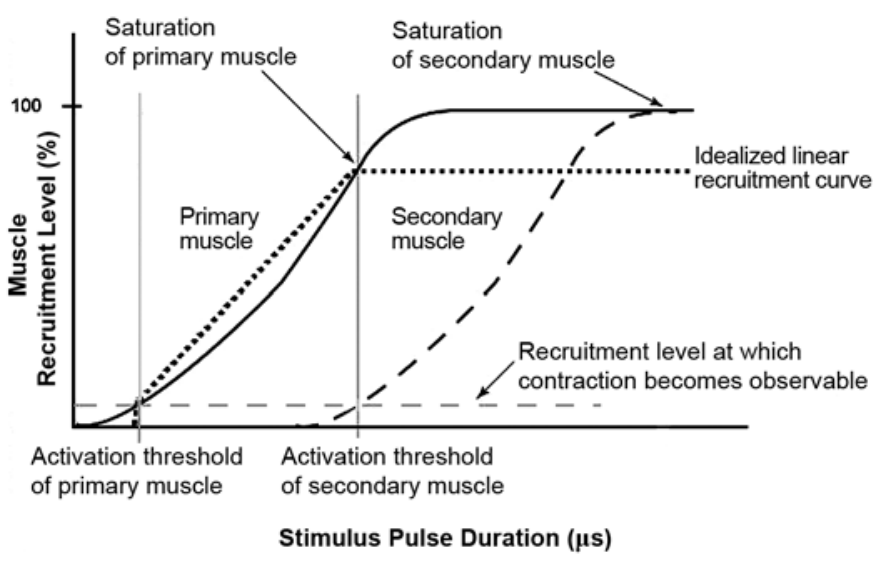

Figure 3.

Idealized linear recruitment curve (black dotted line) derived from threshold and saturation values of stimulus pulse duration (defined as values below which no noticeable contraction from primary muscle exists and above which either no appreciable increase in force production or recruitment of secondary muscle exists, respectively). Gray vertical lines represent activation thresholds of primary and secondary muscles. Solid black line indicates primary muscle activation curve. Black dashed line indicates secondary muscle activation curve. Horizontal, gray, dashed line represents recruitment level at which muscle contraction becomes observable.

trials alternated between starting position leaning backward or leaning forward). The subject was then given as much time as necessary once stimulation was applied (or not applied in the $0 \%$ case) to find a seated balance position with his arms crossed in front of him. After he was able to maintain the posture for $3 \mathrm{~s}$, the position was captured by the camera system. The subject was then instructed to steady himself with his hands and the stimulation was removed.

For each stimulation condition, an average posture was calculated across all collected trials. From the data acquired with the erector spinae electrodes at maximum stimulation (saturation or $100 \%$ of available output), the maximum force output from the stimulated muscle bulk of the erector spinae was determined. This maximum force output was calculated by dividing the active moment required for the subject to maintain the posture (as calculated by inverse dynamics) by the moment arm of the erector spinae in that position.

For the hip muscles, we took origins, insertions, and force-generating capacities from Delp [30-31] as provided in SIMM. Our model included bilateral representations of the semimembranosus and the gluteus maximus for hip extension and the rectus femoris for hip flexion.
These muscles are also accessible by surface [20], intramuscular $[18,21]$, and epimysial $[9,19]$ stimulating electrodes. The muscle lengths and cross-sectional areas of the muscles of interest are shown in Table 4. SIMM represents large muscles with broad origins and insertions, like the gluteus maximus, as groups of muscles with specific lines of action and discrete points of attachment. In this case we assumed that all constituent components of the muscle contracted simultaneously and at the same level of activation.

We calculated passive moments at the hip using the equations proposed by Amankwah et al. [32] for nondisabled subjects and subjects with SCI. For a seated, static case, these simplified to

$$
\mathrm{PT}=13 e^{\left(-0.87 \theta+0.05 \theta_{K}\right) \pi / 180}-6.3 e^{\left(1.3 \theta-0.65 \theta_{K}\right) \pi / 180}
$$

(for nondisabled subjects) and

$$
\mathrm{PT}=22 e^{\left(-1.4 \theta+0.16 \theta_{K}\right) \pi / 180}-0.83 e^{\left(2.9 \theta-1.3 \theta_{K}\right) \pi / 180}
$$

(for subjects with SCI), where PT = passive torque about the hip joint $(\mathrm{N} \cdot \mathrm{m}), \theta=$ hip angle (degrees), and $\theta_{K}=$ knee angle (degrees).

\section{Comparison of Predicted and Experimentally Derived Moments for Subject with Spinal Cord Injury}

The moments required for the subject with SCI to balance himself using different amounts of electrical stimulation of the erector spinae were compared with the outputs of the muscles and passive spine elements predicted by the model. We calculated the moments required for the subject to balance in the different postures by running the experimentally determined joint angles through inverse dynamic calculations using the model, with inertial properties calibrated for the subject with SCI. The joint moments generated by the erector spinae were calculated by multiplying the percentage activation $(0 \%$, $50 \%$, or $100 \%$ ) by the maximum erector spinae output for the subject. The passive torques were calculated for the experimentally determined lumbrosacral and hip joint angles with Equations (2) to (5).

\section{Maximum Stabilization Moments Attainable with Functional Electrical Stimulation}

After inertias and maximum muscle forces were calculated for nondisabled and FES parameters (with maximum FES muscle force output equal to $50 \%$ of 
Table 4.

Length and cross-sectional area for hip muscles. Gluteus maximus has multiple lengths listed because multiple attachments exist on each bony segment.

\begin{tabular}{|c|c|c|}
\hline Muscle & $\begin{array}{c}\text { Length } \\
\text { (cm) }\end{array}$ & $\begin{array}{c}\text { Cross-Sectional } \\
\text { Area }\left(\mathrm{cm}^{2}\right)\end{array}$ \\
\hline Gluteus Maximus & $\begin{array}{l}15.0 \\
13.0 \\
14.0 \\
12.0 \\
17.0 \\
15.0\end{array}$ & 51.8 \\
\hline Rectus Femoris & 86.0 & 12.8 \\
\hline Semimembranosus & 42.0 & 16.9 \\
\hline
\end{tabular}

nondisabled), we used the model to predict the maximum restorative moment that the hip and trunk could develop in response to an externally applied disturbance. For these simulations, the model was positioned in a neutral seated posture and an iteratively growing external force was applied at a point equidistant between the shoulders from $0^{\circ}$ (force from directly behind the model) to $180^{\circ}$ (force from directly in front of the model).

Each level of externally applied moment for each angle was compared with the maximum moments that could be generated by the muscles in that posture. The maximum moment that could be resisted in each direction was then computed and tabulated.

\section{Attainable Postures with Functional Electrical Stimulation}

We also used the model to approximate the range of postures that could be elicited if all the modeled muscles were actuated and no external disturbance forces existed. For these calculations, we assumed that the trunk and hip angles varied linearly with a variation of $1.15^{\circ}$ of trunk flexion for every $1^{\circ}$ of hip flexion as reported in Lee [33]. For each candidate posture, we then determined if the maximum muscle force that the FES-based model could generate would allow that posture to be maintained against gravity. We achieved this by running the activations to the different muscles through a nonlinear optimization routine designed to (if possible) minimize the sum of squares of muscle stress across the full set of muscles, while still keeping all muscles within the level of their maximum force output [34].

\section{RESULTS}

\section{Comparison of Predicted and Experimental Postures for Subject with Spinal Cord Injury}

The average posture and standard deviation for the three different erector spinae stimulation conditions in the SCI experiment are shown in Figure 4. As described in the Methods section (p. 140), we used the position attained when the erector spinae was stimulated at maximum values of pulse duration to approximate the force output of the muscle bulk activated by the electrodes in this subject. This yielded a value $202.6 \mathrm{~N}$ bilaterally. By comparison, nondisabled muscle strength was calculated at $1,562.5 \mathrm{~N}$ bilaterally. If the FES muscle force output is 50 percent of nondisabled, this result implies that only 26 percent of the erector spinae bulk was activated by the electrodes in this subject. Over the small range being observed here, both spine and back position were roughly linearly related to erector spinae activation level.

\section{Comparison of Predicted and Experimentally Derived Moments with Functional Electrical Stimulation}

The hip and back moments needed to stabilize the model at the experimentally measured postures are shown in Table 5. This table also shows the calculated muscle and passively generated moments at those same

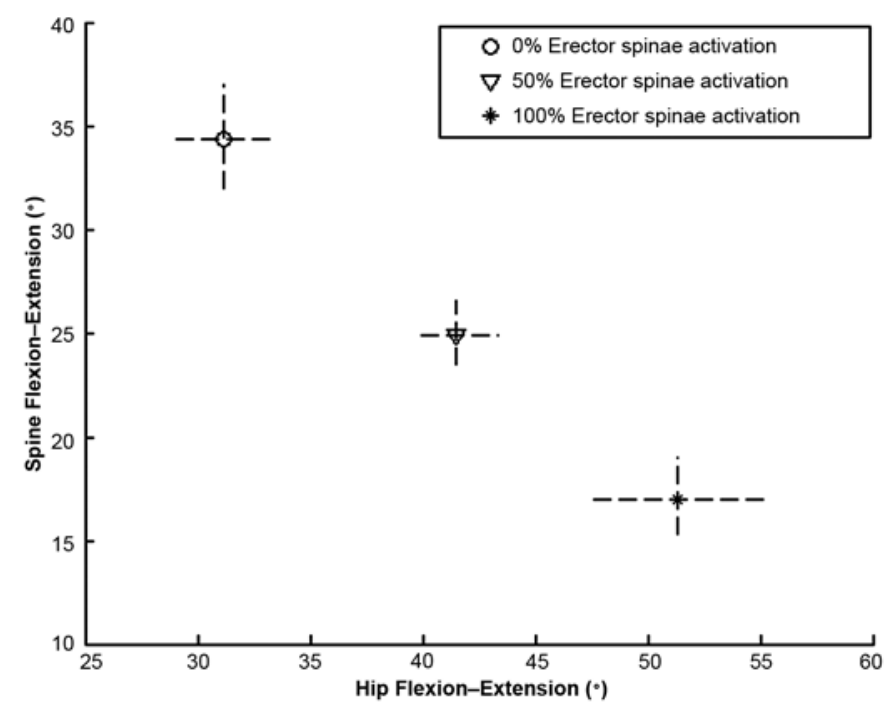

Figure 4.

Mean hip and trunk angles (with standard deviations, dashed lines) from spinal cord injury experiment for $0 \%, 50 \%$, and $100 \%$ electrical activation of erector spinae. Hip and spine angles are defined as zero when standing. Hip flexion and spine flexion are in positive direction. 
Table 5.

Differences between experimentally determined moments required to maintain postures and moments generated by muscles and passive structures for back and hip as predicted by simulations modified for experimental subject. Positive moments act to flex given joint.

\begin{tabular}{ccccccc}
\hline $\begin{array}{c}\text { Stimulation } \\
\text { Coordination (\%) }\end{array}$ & $\begin{array}{c}\text { Experimental } \\
\text { Back (N·m) }\end{array}$ & $\begin{array}{c}\text { Predicted Back } \\
\mathbf{( N \cdot m )}\end{array}$ & $\begin{array}{c}\Delta \text { Back } \\
\mathbf{( N \cdot m )}\end{array}$ & $\begin{array}{c}\text { Experimental Hip } \\
\mathbf{( N \cdot m )}\end{array}$ & $\begin{array}{c}\text { Predicted Hip } \\
\mathbf{( N \cdot m )}\end{array}$ & $\begin{array}{c}\Delta \text { Hip } \\
\mathbf{( N \cdot m )}\end{array}$ \\
\hline 0 & -3.0 & -4.9 & 1.9 & 14.0 & 12.2 & 1.8 \\
50 & -8.4 & -10.0 & 1.6 & 2.0 & 8.9 & -6.9 \\
\hline \hline
\end{tabular}

experimental postures. The calculated value for the 100 percent condition is not shown because we used it to determine the trunk muscle-force output for this subject (therefore the calculated trunk muscle output and passive moments are by definition identical to the moment required to stabilize the model in that position). For the 0 percent condition, a good correlation existed between the required and calculated moment for both the hip and trunk, with experimentally determined values within $2 \mathrm{~N} \cdot \mathrm{m}$. For the 50 percent condition, an excellent correlation existed between required and calculated moment for the trunk $(<2 \mathrm{~N} \cdot \mathrm{m})$, but predicted and experimental values for the hip joints deviated to a greater extent $(\sim 7 \mathrm{~N} \cdot \mathrm{m}$ difference). The error for the hip joint is greater in the model possibly because the hip supports the weight of the trunk and any errors are magnified, whereas the trunk only supports itself.

\section{Maximum Stabilization Moments Attainable with Functional Electrical Stimulation}

Using the techniques described in the Methods section (p. 140), we calculated the maximum predicted restorative moment that could be applied to an external disturbance assuming 50 percent of nondisabled maximum force output of the muscles, which simulated the situation for paralyzed muscle activated with FES. These data are shown in Figure 5 for the $180^{\circ}$ of space to the model's right side. Because the model is symmetric about the sagittal plane, the left-sided maximum moments are the mirror images of those presented in the figure. The FES model shows a maximum restoration moment of 25 to $45 \mathrm{~N} \cdot \mathrm{m}$ across the workspace. The largest available restoration moments were noted when the model was leaning directly forward or directly backward, where the powerful erector spinae and rectus abdominis could be used fully. Maximum available restorative moments with FES are 42 and $48 \mathrm{~N} \cdot \mathrm{m}$ in the anterior and posterior directions, respectively and $28 \mathrm{~N} \cdot \mathrm{m}$ in the medial-lateral direction. Notably, although the erector spinae can contribute to restoration moment in the lateral direction, only half of the muscle bulk can be used for this purpose. Also, the erector spinae has a small moment arm for lateral moments.

\section{Feasible Postures Attainable with Functional Electrical Stimulation}

Figure 6 illustrates the range of seated postures that would be feasible with full activation of the muscles modeled (i.e., nondisabled case) and FES activation of the muscles modeled given the conditions described in the Methods section (p. 140) (i.e., at best 50\% of nondisabled maximum force output).

The ranges of motion predicted for nondisabled individuals by the model coincide with published data from the literature. Specifically, Lee and Wong measured 20 nondisabled subjects while they were leaning forward and sideways with an average maximum forward bend of $58^{\circ}$ at the $\mathrm{L}$ spine and $56^{\circ}$ at the hip [33]. They also measured an average maximum lateral bend of $20.5^{\circ}$ at the $\mathrm{L}$ spine. Although not a perfect match because of the different experimental conditions and the reduced muscle set in our model simulations, these data still correspond well with the predictions made with the nondisabled model parameters, which show a maximum forward bend at the spine of $64^{\circ}$, a maximum forward bend at the hip of $58^{\circ}$, and a maximum lateral bend at the spine of $20^{\circ}$.

Note that this experiment simulated a particular set of hip-trunk angles that mimic biological behavior, whereas in theory any relationship between the two angles could be specified arbitrarily. For this configuration, the FES simulation shows a forward leaning range of about $13^{\circ}$ at the hips and $15^{\circ}$ at the trunk and a backward leaning range of about $9^{\circ}$ at the hips and $10^{\circ}$ at the trunk (measured from a neutral posture). The trunk has a lateral bending range of about $9^{\circ}$ maximum. The lateral range decreases significantly as the model leans forward and imbalances between left and right musculature cannot be maintained while still providing sufficient extension against gravity. These simulations indicate that the possibility exists to stabilize the trunk and pelvis sufficiently with FES that up to 51 percent of nondisabled leaning range of motion in 
the sagittal plane and 41 percent of nondisabled lateral bending could be obtained. In general, the extent of the posture space in the coronal plane theoretically possible with FES is 28 percent of that predicted with all of the available muscle force in the nondisabled case.

Figure 7 shows the predicted percentage of full activations (for the FES model) of the different muscles for

\section{$180^{\circ}$}

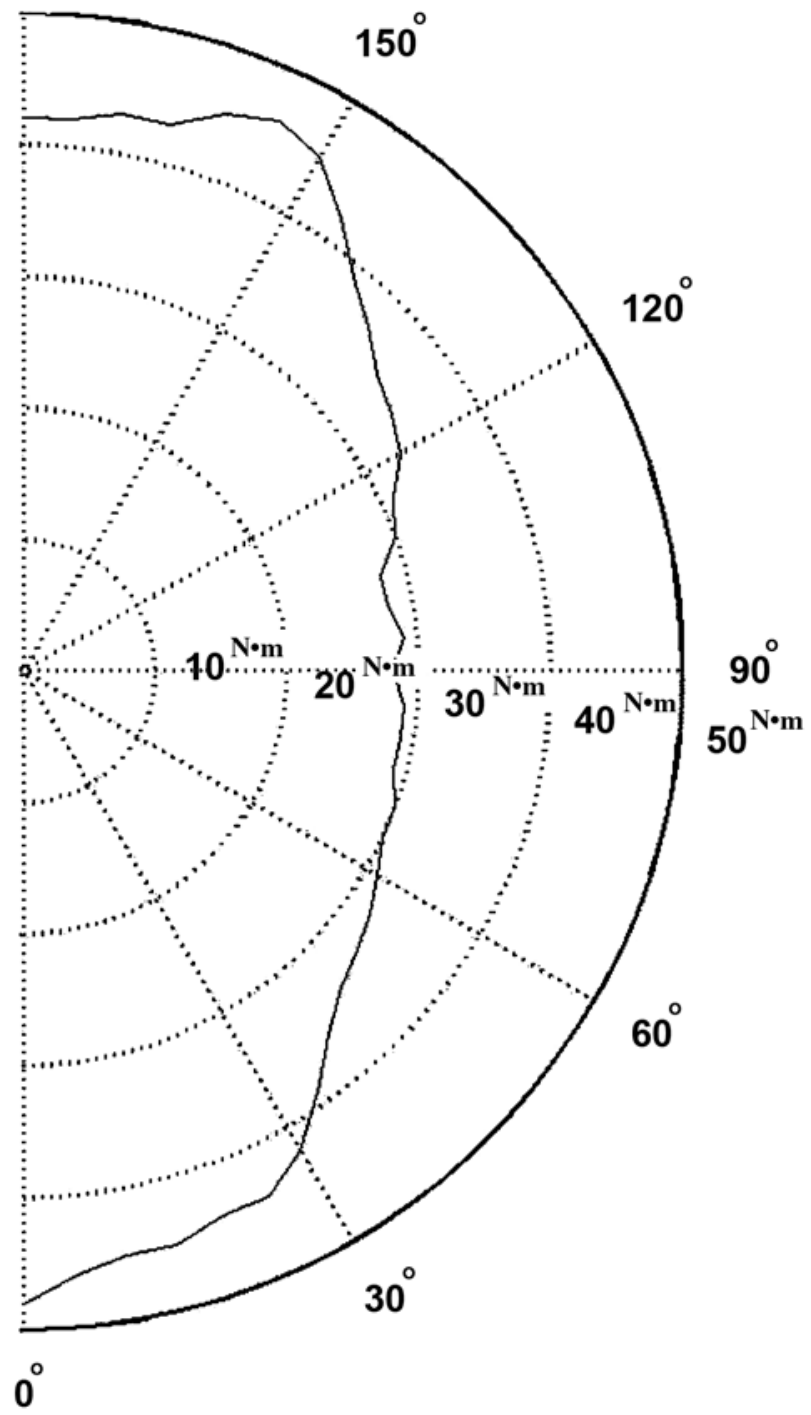

Figure 5.

Maximum torque (in newton meters) that can be generated in each direction in model's transverse plane (from neutral seated posture) by simulated electrical activation of paralyzed muscles in response to a disturbance torque. $0^{\circ}$ represents force pushing from directly behind midpoint between shoulders and $180^{\circ}$ represents force pushing from front. the seated postures in the sagittal plain (no lateral bending) shown in Figure 6. Since the optimization routine we used minimizes muscle stress, the larger erector spinae are almost fully activated before the smaller quadratus are recruited for forward-leaning postures. At slightly higher required moments, the quadratus lumborum is rapidly recruited to make up the difference. The trunk and hip flexor muscles (rectus abdominis and rectus femoris) are used only for leaning backward from neutral. The posture in the sagittal plane for which most of the forces go to zero is similar to the neutral position measured with the experimental subject with SCI (between $30^{\circ}$ and $40^{\circ}$ of forward-trunk flexion).

\section{DISCUSSION}

The results indicate that a useful system for maintaining and altering seated posture could be implemented with the described muscles under FES control. The simulations predict that such a system could provide 25 to $45 \mathrm{~N}$ of restorative moment in response to force disturbances applied to the trunk. Conceptually, this performance is equivalent to holding a $5-10 \mathrm{lb}$ weight at arms length, which has profound functional implications for

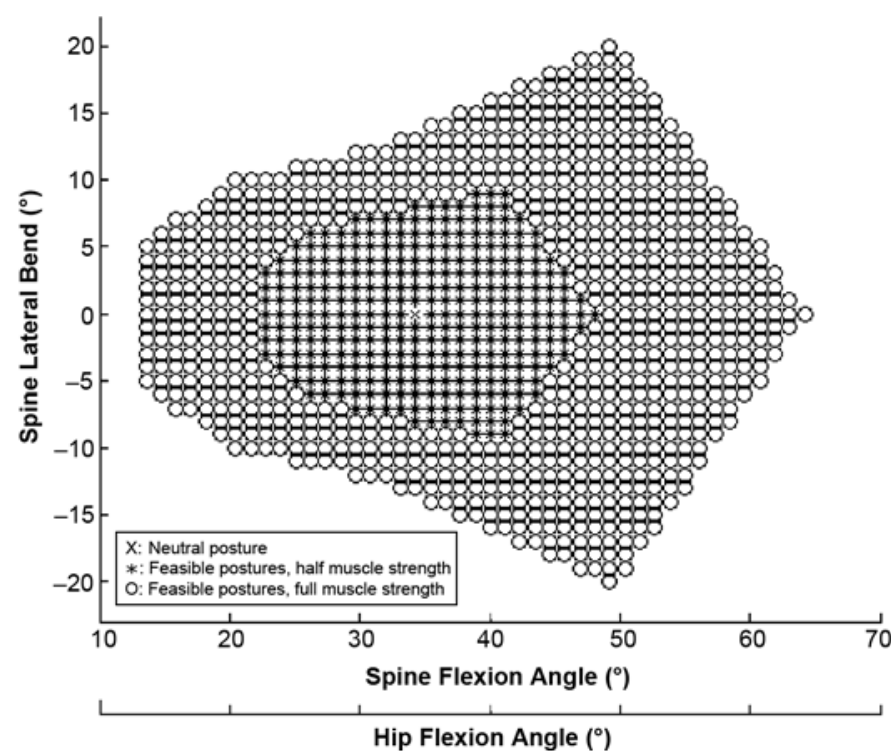

Figure 6.

Feasible seated postures with full muscle strength and half muscle strength postulated by biomechanical model that assumes fixed ratio between hip and trunk angular excursions. 


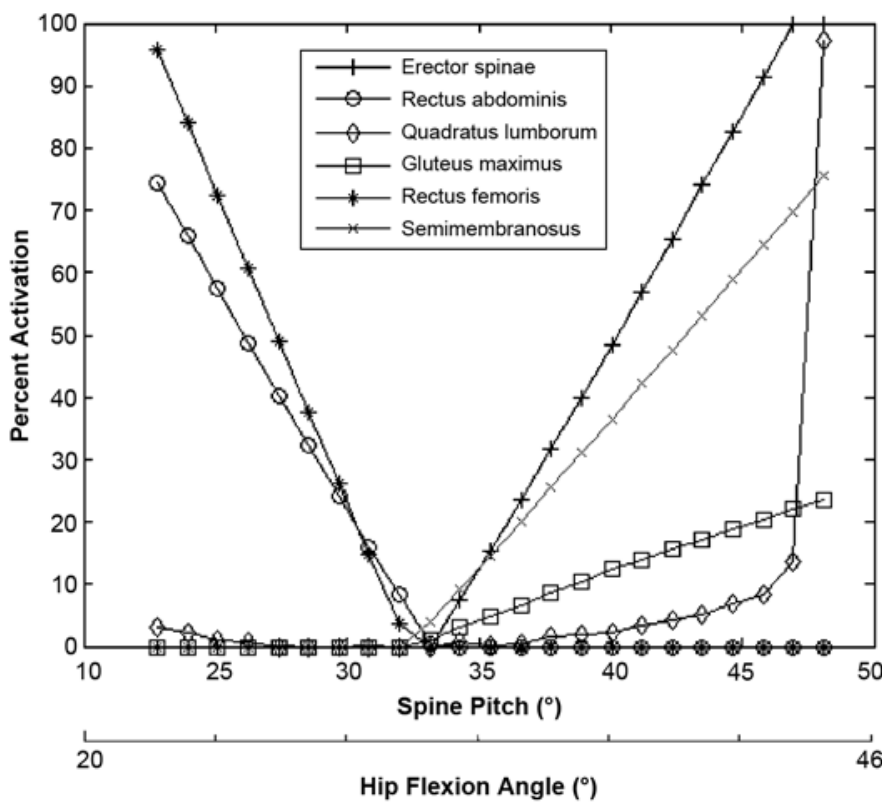

Figure 7.

Percentage of predicted maximum activation of muscle for different trunk and hip muscles used to maintain different postures in sagittal plane.

individuals with SCI who often struggle with simple activities of daily living.

The maximum feasible posture simulation predicts that approximately $28^{\circ}$ of combined hip-trunk flexion forward from a neutral posture may be possible with FES. This corresponds to about $0.25 \mathrm{~m}$ of increased workspace at the level of the shoulder. The model also predicts that about $9^{\circ}$ of lateral movement that would otherwise be unavailable to a person with SCI could be possible with FES, which would further expand the workspace and improve the functional independence of FES system users.

Although these theoretical results are encouraging and support the feasibility of FES for controlling seated posture and trunk position, a major obstacle to the development of a practical system that achieves this level of performance is obtaining sufficient stimulated force output of the trunk musculature. The results of this study indicate that for best control of hip-trunk position, stimulating electrodes need to more completely recruit the targeted muscles. Force output from electrically stimulated muscle is a function of several factors, including stimulation waveform and parameters, muscle atrophy, fiber type, and electrode location relative to the targeted neural structures. Clearly, from our results, the single intramus- cular electrode located at the T12-L1 spinal roots does not fully activate the entirety of the erector spinae muscle mass. Our simulations indicate that our subject with SCI was using about 26 percent of his potentially available muscle mass at maximum levels of stimulation. This is unsurprising given that the erector spinae are segmentally innervated and the current system has only a single pair of electrodes at the L region. Clearly fibers of the erector mass that are innervated from different vertebral levels are not activated in this case. The result indicating that only 26 percent of the available muscle force was being recruited is reasonable given that the spinal root at T12L1 innervates a similar fraction of the $\mathrm{L}$ erector mass. Multiple electrodes distributed over a number of segmental levels would be one possible way of more fully activating the target muscles.

Several assumptions were made in the creation of the model employed in our analysis. In the FES model, we assumed that a maximum of 50 percent of nondisabled force could be generated by fully recruiting the entire paralyzed muscle. Although the majority of data supporting this maximum percentage is in lower-limb muscle literature, no reasons exist that it would be significantly different for trunk muscle. Also, we assumed that the passive elasticity of the spine was linear over the range of motion in question and with multiple muscle activations. This is certainly not true [15,35-37], but since the values involved were small, the assumption is unlikely to have largely affected the results. Furthermore, since the passive elasticity is likely to increase at large angles and large loads, this effect is unlikely to decrease the available workspace.

We also assumed that controlling moments about the L5-S1 joint would be sufficient to control trunk posture without independently actuating other spinal level musculature. While the method of natural control of back posture is still unclear, the fact that a single set of electrodes in our experimental subject led to stable seated postures supports this assumption.

\section{CONCLUSIONS}

Computer simulations and experimental results indicate that control of seated posture via coordinated FES of the hip and trunk musculature in individuals with paralysis from SCI is feasible and may lead to improved workspace, stability, and the ability to reject disturbances. A 
physiologically reasonable and anatomically based biomechanical model of the hips and torso was constructed, which yielded predictions in good agreement with the experimental data. Analyses with the model indicate that implanted FES systems with bilateral intramuscular electrodes at the T12-L1 spinal roots appear to recruit approximately one-quarter of the trunk extension force expected by FES because of the segmental nature of the innervations of the erector spinae. Practical FES systems for stabilizing the torso and hips may need to include electrodes and independent stimulus channels at multiple vertebral segments. The simulations also indicate that an FES system that includes the erector spinae, quadratus lumborum, rectus abdominis, gluteus maximus, and semimembranosus muscles can control the hip and trunk and lead to improved disturbance rejection, which potentially enhances a subject's ability to manipulate objects or perform bimanual tasks without loss of balance. Furthermore, activation of these muscles can increase the range of available postures for seated individuals with SCI and thus expand their access to and control of the environment. Although these results remain to be verified experimentally with subjects equipped with the full set of muscles described, significant changes in posture were achieved experimentally by applying various levels of stimulation to only the $\mathrm{L}$ erector spinae.

\section{ACKNOWLEDGMENTS}

We particularly appreciate the contributions of the implanted FES system user who volunteered for these experiments.

This material was based on work supported by the National Institute of Neurological Disorders and Stroke/ National Institutes of Health (postdoctoral training grant 1F32NS44700-01), the National Institute of Neurological Diseases and Stroke (grant R01-NS-40547), and the Cleveland FES Center, a Rehabilitation Research and Development Service Center of Excellence of the VA.

The authors have declared that no competing interests exist.

\section{REFERENCES}

1. Triolo RJ, Bieri C, Uhlir J, Kobetic R, Scheiner A, Marsolais EB. Implanted functional neuromuscular stimulation systems for individuals with cervical spinal cord injuries:
Clinical case reports. Arch Phys Med Rehabil. 1996; 77(11):1119-28. [PMID: 8931521]

2. Sharma M, Marsolais EB, Polando G, Triolo RJ, Davis JA Jr, Bhadra N, Uhlir JP. Implantation of a 16-channel functional electrical stimulation walking system. Clin Orthop Relat Res. 1998;(347):236-42. [PMID: 9520896]

3. Kobetic R, Triolo RJ, Uhlir JP, Bieri C, Wibowo M, Polando G, Marsolais EB, Davis JA Jr, Ferguson KA. Implanted functional electrical stimulation system for mobility in paraplegia: A follow-up case report. IEEE Trans Rehabil Eng. 1999;7(4):390-98. [PMID: 10609626]

4. Keith MW. Neuroprostheses for the upper extremity. Microsurgery. 2001;21(6):256-63. [PMID: 11746557]

5. Marsolais EB, Kobetic R, Polando G, Ferguson KA, Tashman S, Gaudio R, Nandurkar S, Lehneis HR. The Case Western Reserve University hybrid gait orthosis. J Spinal Cord Med. 2000;23(2):100-108. [PMID: 10914350]

6. Chen CF, Lien IN, Wu MC. Respiratory function in patients with spinal cord injuries: Effects of posture. Paraplegia. 1990;28(2):81-86. [PMID: 2235026]

7. Walter JS, Sacks J, Othman R, Rankin AZ, Nemchausky B, Chintam R, Wheeler JS. A database of self-reported secondary medical problems among VA spinal cord injury patients: Its role in clinical care and management. J Rehabil Res Dev. 2002;39(1):53-61. [PMID: 11926327]

8. Curtis KA, Kindlin CM, Reich KM, White DE. Functional reach in wheelchair users: The effects of trunk and lower extremity stabilization. Arch Phys Med Rehabil. 1995; 76(4):360-67. [PMID: 7717837]

9. Davis JA Jr, Triolo RJ, Uhlir J, Bieri C, Rohde L, Lissy DJ. Preliminary performance of a surgically implanted neuroprosthesis for standing and transfers-Where do we stand? J Rehabil Res Dev. 2001;38(6):609-17. [PMID: 11767968]

10. Wilkenfeld AJ, Triolo RJ, Audu M. A model for simulating dynamic control of seated trunk posture using functional electrical stimulation [abstract]. J Spinal Cord Med. 2003; 23 (Suppl) 1:S15.

11. Kukke SN, Triolo RJ. The effects of trunk stimulation on bimanual seated workspace. IEEE Trans Neural Syst Rehabil Eng. 2004;12(2):177-85. [PMID: 15218932]

12. Cholewicki J, McGill SM. Mechanical stability of the in vivo lumbar spine: Implications for injury and chronic low back pain. Clin Biomech (Briston, Avon). 1996;11(1):1-15. [PMID: 11415593]

13. Stokes IA, Garder-Morse M. Lumbar spine maximum effort and muscle recruitment patterns predicted by a model with multijoint muscles and joints with stiffness. J Biomech. 1995;28(2):173-86. [PMID: 789860]

14. Daggfeldt K. Biomechanics of back extension torque production about the lumbar spine [dissertation]. Stockholm (Sweden): Department of Neuroscience, Karolinska Institute; 2002. p. 1-42. 
15. White AA, Panjabi MM. Clinical biomechanics of the spine. 2nd ed. Philadelphia (PA): Lippincott Williams \& Wilkins; 1990. p. 46-49; 85-125.

16. McGill SM. A myoelectrically based dynamic threedimensional model to predict loads on lumbar spine tissues during lateral bending. J Biomech. 1992;25(4):395-414. [PMID: 1533860]

17. Winter DA. Biomechanics and motor control of human movement. 2nd ed. Toronto (Canada): John Wiley \& Sons, Inc; 1990. p. 56-57.

18. Triolo RJ, Liu MQ, Kobetic R, Uhlir J. Selectivity of intramuscular stimulating electrodes in the lower limbs. J Rehabil Res Dev. 2001;38(5):533-44. [PMID: 11732831]

19. Davis JA Jr, Triolo RJ, Uhlir J, Bhadra N, Lissy DA, Nandurkar S, Marsolais EB. Surgical technique for installing an 8-channel neuroprosthesis for standing. Clin Orthop Relat Res. 2001;(385):237-52. [PMID: 11302320]

20. Baker LL, McNeal DR, Benton LA, Bowman BR, Waters RL. Neuromuscular electrical stimulation-A practical clinical guide. 3rd ed. Downey (CA): Rehabilitation Engineering Center; 1993. p. 141-68.

21. Marsolais EB, Kobetic R. Implantation techniques and experience with percutaneous intramuscular electrodes in the lower extremities. J Rehabil Res Dev. 1986;23(3):1-8. [PMID: 3490566]

22. Marsolais EB, Kobetic R. Development of a practical electrical stimulation system for restoring gait in the paralyzed patient. Clin Orthop Relat Res. 1988;(233):64-74. [PMID: 3261221]

23. Kobetic R, Marsolais EB. Control of paraplegic gait by detection of discrete events. Proceedings of the International Conference of the Association for the Advancement of Rehabilitation Technology; 1988 Jun 25-30; Montreal, Canada. Washington (DC): RESNA; 1988. p. 346-47.

24. Delp SL, Suryanarayanan S, Murray WM, Uhlir J, Triolo RJ. Architecture of the rectus abdominis, quadratus lumborum, and erector spinae. J Biomech. 2001;34(3):371-75. [PMID: 11182129]

25. Tracy M, Gibson MJ, Szypryt EP, Rutherford A, Corlett EN. The geometry of the muscles of the lumbar spine determined by magnetic resonance imaging. Spine. 1989; 14(2):186-93. [PMID: 2922639]

26. Granata KP, Marras WS. An EMG-assisted model of trunk loading during free-dynamic lifting. J Biomech. 1995; 28(11):1309-17. [PMID: 8522544]
27. Keller TS, Roy AL. Posture-dependent isometric trunk extension and flexion strength in normal male and female subjects. J Spinal Disord Tech. 2002;15(4):312-18.

[PMID: 12177548]

28. Kobetic R, Marsolais EB, Miller PC. Function and strength of electrically stimulated hip flexor muscles in paraplegia. IEEE Trans Rehabil Eng. 1994;2(1):11-17.

29. Carrol SG, Triolo RJ, Chizeck HJ, Kobetic R, Marsolais EB. Tetanic responses of electrically stimulated paralyzed muscle at varying interpulse intervals. IEEE Trans Biomed Eng. 1989;36(7):644-53. [PMID: 2787276] Erratum in: IEEE Trans Biomed Eng. 1989;36(11):1143.

30. Delp SL. Surgery simulation: A computer graphics system to analyze and design musculoskeletal reconstructions of the lower limb [dissertation]. Stanford (CA): Department of Mechanical Engineering, Stanford University; 1990. p. 1-128.

31. Delp SL, Loan JP, Hoy MG, Zajac FE, Topp EL, Rosen JM. An interactive graphics-based model of the lower extremity to study orthopaedic surgical procedures. IEEE Trans Biomed Eng. 1990;37(8):756-67. [PMID: 2210784

32. Amankwah K, Triolo RJ, Kirsch R. Effects of spinal cord injury on lower-limb passive joint moments revealed through a nonlinear viscoelastic model. J Rehabil Res Dev. 2004;41(1):15-32. [PMID: 15273894]

33. Lee RY, Wong TK. Relationship between the movements of the lumbar spine and hip. Hum Mov Sci. 2002;21(4): 481-94. [PMID: 12450680]

34. Lawrence CT, Zhou JL, Tits AL. User's guide for CFSQP version 2.5: A C code for solving (large scale) constrained nonlinear (minimax) optimization problems, generating iterates satisfying all inequality constraints. Technical report TR-94-16r1. College Park (MD): Institute for Systems Research, University of Maryland; 1997. p. 1-44.

35. Miller JA, Schultz AB, Warwick DN, Spencer DL. Mechanical properties of lumbar spine motion segments under large loads. J Biomech. 1986;19(1):79-84. [PMID: 3949818$]$

36. Edwards WT, Hayes WC, Posner I, White AA 3rd, Mann RW. Variation of lumbar spine stiffness with load. J Biomech Eng. 1987;109(1):35-42. [PMID: 3560877]

37. Janevic J, Ashton-Miller JA, Schultz AB. Large compressive preloads decrease lumbar motion segment flexibility. J Orthop Res. 1991;9(2):228-36. [PMID: 1992073]

Submitted for publication June 16, 2005. Accepted in revised form November 4, 2005. 